Journal of Engineering and Applied Sciences 14 (19): 7165-7173, 2019

ISSN: 1816-949X

(C) Medwell Journals, 2019

\title{
Comparison Between the Analytical and Monte Carlo Simulation Solutions for Two-Dimensional Ising Model of a Ferromagnet
}

\author{
F.F. Jurado-Lasso, N. Jurado-Lasso, J.S Baena-Vasquez and J.F. Jurado \\ Propiedades Termicas Dielectricas de Compositos Research Group, Department of Basic Sciences, \\ Universidad Nacional de Colombia, Palmira, Colombia
}

\begin{abstract}
The response to the temperature for a two-Dimensional (2D) spin array of a lattice of $2 \times 2$ size Ising-Onsager type was compared with the exact solution and simulation in the formalism of the Monte Carlo simulation. The functions energy, magnetization, heat capacity and susceptibility per spin determined by Monte Carlo simulation and the Metropolis algorithm, showed dispersion of close to $1 \%$ when compared to the exact solution and the Onsager model. The finite scaling method for $2 \mathrm{D}$ lattices of $2 \times 2,4 \times 4,8 \times 8,16 \times 16,32 \times 32$ and $64 \times 64$ size, allowed the calculation of the critical exponents of scaling function magnetization, specific heat capacity and susceptibility. The results show that the scaling functions are independent of lattice size but dependent on temperature and the critical exponent (class of universality).
\end{abstract}

Key words: Ising model, Onsager model, Monte Carlo simulation, scaling method, scaling function, critical exponent

\section{INTRODUCTION}

Ising model two-dimensional: onsager: Insome materials, long-distance exchange interaction between magnetic moments plays an important role in terms of magnetic and charge carrier properties. The interaction is measured by a constant known as the exchange constant. Many models and formalism have been developed help reach a conclusive understanding of this phenomenology. Among them, we highlight the design of a preferably symmetric, distribution of atoms known as the spin model in which each lattice point is associated to the spin variable. The atomic arrangement may involve different types of magnetism which converge into two large groups cooperative and non-competitive (Binner et al., 2006). The first group is characterized by the fact that it presents no interaction between the magnetic spins. For the second group, the main type of interaction is associated to the interaction between spins. An example of the latter is the arrangement presented in ferromagnetism and antiferromagnetism. Many proposals have been put forward to explain ferromagnetism among them, the, so, called Ising model. In its first version, proposed as part of a thesis by Wilhelm Lenz (1920), Brush (1967), the model was presented for a one-dimensional spin array. A few years later, the model was presented more formally in the research of Ernst Ising in 1925 (Onsager, 1943) with one of its most relevant results as the non-existence of phase transi tions in one-dimensional arrays. Subsequently, Onsager (1943) and (Kaufman, 1949) presented the solution for a two-dimensional (2D) spin array. The most relevant contribution of this research was the evidence it provided of a phase transition marked by a transition temperature. The Ising model was conceived to explain how some materials can present a magnetic order as a consequence of magnetic interaction (ferromagnetic or antiferromagnetic) even in the absence of an external applied magnetic field. The effect can be observed below a temperature value (Tc and $\mathrm{Tn}$ ), respectively. Above this temperature for both cases, the individual spins would be thermally disordered pointing in arbitrary directions and resulting in a net magnetization of zero. In the finite spin configuration for $2 \mathrm{D}(\mathrm{N}$ rows and $\mathrm{N}$ columns), the input hypothesis to determine an observable through a method that involves recurrence relations is based on the boundary conditions. That is it involves integration with previous chains ( $\mu$-th row) (vertical dependence) and the previous spins $\mathrm{S}_{\mathrm{i}-1}$ (horizontal dependence). For example, for the set of spins in row $\mu=S_{1}, S_{2}, S_{3}, \ldots, S_{N}$, the boundary conditions of the spin configuration are limited by $\mu_{\mathrm{N}+1}=\mu_{1}$ y $\mathrm{S}_{\mathrm{N}+1}=\mathrm{S}_{1}$. Where the energy of the interaction between rows $\mu$ and $\mu^{\prime}$ :

$$
\mathrm{E}\left(\mu_{\alpha}, \mu_{\alpha}^{\prime}\right)=-\mathrm{j} \sum_{\mathrm{k}=1}^{\mathrm{N}} \mathrm{S}_{\mathrm{k}} \mathrm{S}_{\mathrm{k}}^{\prime}
$$

Corresponding Author: J.F. Jurado, Propiedades Termicas Dielectricas de Compositos Research Group, Department of Basic Sciences, Universidad Nacional de Colombia, Palmira, Colombia 
$\mathrm{j}$ being the constant interaction between nearest neighbours. In the case of the presence of an external field $h$, the energy of each is given by:

$$
\mathrm{E}_{\mathrm{i}}(\mu)=\mathrm{j} \sum_{\mathrm{k}=1}^{\mathrm{N}} \mathrm{S}_{\mathrm{k}} \mathrm{S}_{\mathrm{k}+1}-\mathrm{h} \sum_{\mathrm{k}=1}^{\mathrm{N}} \mathrm{S}_{\mathrm{k}}
$$

The total energy:

$$
E_{t}(\mu)=\sum_{\alpha=1}^{N}\left[E\left(\mu_{\alpha}, \mu_{\alpha+1}\right)+E\left(\mu_{\alpha}\right)\right]
$$

In these case we will only consider classical systems and mainly focus on the canonical ensemble where the partition function is give as $\mathrm{z}$ and the summation running over all possible state of the system:

$$
\begin{gathered}
\mathrm{z}(\mathrm{h}, \mathrm{T})=\sum_{\text {allstates }} \mathrm{e}^{-\beta \mathrm{E}_{\mathrm{i}}} \mathrm{z}(\mathrm{h}, \mathrm{T})=\sum_{\mu 1} \sum_{\mu 2} \sum_{\mu 3} \\
\sum_{\mu_{\mathrm{N}}} \mathrm{e}^{-\beta} \sum_{\alpha_{1}\left[\mathrm{E}\left(\mu_{\alpha,}, \mu_{\alpha+1}\right)+\mathrm{E}\left(\mu_{\alpha}\right)\right]}^{\mathrm{N}}
\end{gathered}
$$

When considering each exponential term as a matrix element $\mathrm{P}$. The partition function can be expressed in terms of trace $\mathrm{P}^{\mathrm{N}}, \mathrm{P}$ being of the order $2^{\mathrm{N}} \times 2^{\mathrm{N}}$. The matrix trace is invariant under transformation. The partition function in terms of eigenvalues:

$$
\begin{gathered}
<\mu_{\alpha}|\mathrm{P}| \mu_{\alpha+1}>=\mathrm{e}^{-\beta\left[\mathrm{E}\left(\mu_{\alpha}, \mu_{\alpha+1}\right)+\mathrm{E}\left(\mu_{\alpha}\right)\right]} \\
\mathrm{z}(\mathrm{h}, \mathrm{T})=\sum_{\mu 1}<\mu_{1}|\mathrm{P}| \mu_{2}>\sum_{\mu 2}<\mu_{2}|\mathrm{P}| \mu_{3}> \\
\sum_{\mu 3}<\mu_{3}|\mathrm{P}| \mu_{4}>, \ldots, \sum_{\mu_{\mathrm{N}}}<\mu_{\mathrm{N}}|\mathrm{P}| \mu_{1}> \\
\mathrm{z}(\mathrm{h}, \mathrm{T})=\sum_{\mu 1}<\mu_{1}\left|\mathrm{P}^{\mathrm{N}}\right| \mu_{1}>=\mathrm{T}_{\mathrm{r}} \mathrm{P}^{\mathrm{N}} \\
\mathrm{z}(\mathrm{h}, \mathrm{T})=\sum_{\alpha=1}^{2^{\mathrm{N}}} \lambda_{\alpha}^{\mathrm{N}}
\end{gathered}
$$

Eigenvalues:

$$
\lambda_{1}>\lambda_{2}>\lambda_{3}>, \ldots, \lambda_{2^{\mathrm{N}}}
$$

The partition function has to be delimited:

$$
\begin{aligned}
& \lambda_{\max }^{\mathrm{N}} \leq \mathrm{z}(\mathrm{h}, \mathrm{T}) \leq 2^{\mathrm{N}} \lambda_{\text {max }}^{\mathrm{N}} \\
& \frac{1}{\mathrm{~N}}\left|\mathrm{n} \lambda_{\max } \leq \frac{1}{\mathrm{~N}^{2}}\right| \mathrm{n}(\mathrm{z}(\mathrm{h}, \mathrm{T})) \leq \frac{1}{\mathrm{~N}} \lambda_{\max }+\frac{1}{\mathrm{~N}} \mid \mathrm{n} 2
\end{aligned}
$$

Based on this last expression and extrapolating the system to the thermodynamic limit, it is possible to determine the terms $\ln \lambda$. This term was found by Onsager in his research and it is known as the Onsager partition function (Newman and Barkema, 1996; Kaufman, 1949; Majumdar, 1966):

$$
\begin{aligned}
& \mid \mathrm{n} \lambda=-\operatorname{nn}(2 \cos (2 \mathrm{~K})) \\
& -\frac{1}{\pi} \int_{0}^{\frac{\pi}{2}} \mathrm{~d} \phi \mid \mathrm{n}\left[\frac{1}{2}\left\{1+\left(1-\mathrm{k}^{2} \sin ^{2} \phi\right)^{\frac{1}{2}}\right\}\right]
\end{aligned}
$$

being $\mathrm{k}=2 \sinh 2 \mathrm{~K} / \cosh ^{2} 2 \mathrm{~K}$ y $\mathrm{K}=\beta \mathrm{j}$. Is the Helmholtz free energy in terms of the partition function:

$$
\mathrm{F}=-\frac{1}{\beta} \operatorname{In} \mathrm{z}
$$

where, the free energy per particle:

$$
f=\frac{F}{N}
$$

The energy per particle in terms of the Onsager term:

$$
\begin{aligned}
& E(T)=-\frac{\partial}{\partial \beta} \ln z=-N \frac{\partial}{\partial \beta} \mid n \lambda \\
& E(T)=-2 j \tanh 2 K+\frac{k}{2 \pi} \frac{d k}{d \beta} \int_{0}^{\pi} d \phi \frac{\sin ^{2} \phi}{\Delta(1+\Delta)}
\end{aligned}
$$

where, $\Delta=\sqrt{1-\mathrm{k}^{2} \sin ^{2} \phi}$ by performing certain operations, we can obtain the energy:

$$
E(k T)=-j \operatorname{coth} 2 K\left(1-\frac{21}{\pi} K_{1}(k)\right)
$$

where, $1=1-2 \tanh ^{2} 2 \mathrm{~K}$ y $\mathrm{K}_{1}(\mathrm{k})=\int_{0}^{\frac{\pi}{2}} \frac{\mathrm{d} \phi}{\sqrt{1-\mathrm{k}^{2} \sin ^{2} \phi}}, \mathrm{K}_{1}(\mathrm{k})$ is the complete elliptic integral of the first kind. The constant field heat capacity:

$$
\begin{gathered}
\mathrm{C}(\mathrm{kT})=\frac{\partial \mathrm{E}}{\partial \mathrm{T}}=-\frac{1}{\mathrm{kT} \mathrm{T}^{2}} \frac{\partial \mathrm{E}}{\partial \beta}=\frac{\mathrm{N}}{\mathrm{kT}^{2}} \frac{\partial^{2} \mid \mathrm{n} \lambda}{\partial \beta^{2}} \\
\mathrm{C}(\mathrm{kT})=\frac{2 \mathrm{k}}{\pi}(\beta \mathrm{j} \operatorname{coth} 2 \mathrm{~K})^{2}\left[2 \mathrm{~K}_{1}(\mathrm{k})-\right. \\
\left.2 \mathrm{E}_{1}(\mathrm{k})-(1-1)\left(\frac{\pi}{2}+1 \mathrm{~K}_{1}(\mathrm{k})\right)\right]
\end{gathered}
$$

$E_{1}(k)$ is the elliptic integral of second kind and is given by Huang (1987):

$$
\mathrm{E}_{1}(\mathrm{k})=\int_{0}^{\frac{\pi}{2}} \sqrt{1-\mathrm{k}^{2} \sin ^{2} \phi} \mathrm{d} \phi
$$

Calculating the elliptic integral must be carried out with care and to facilitate this, it is carried out in the neighborhood of $\mathrm{k}=1$. In these conditions, $\sinh 2 \mathrm{k}_{\mathrm{c}}=1 \mathrm{y}$ 
$\cosh 2 \mathrm{k}_{\mathrm{c}}=\sqrt{1+\sinh ^{2} 2 \mathrm{~K}}=\sqrt{2}$ which implies, $\lim _{\mathrm{T} \rightarrow \mathrm{T}_{\mathrm{c}}}(\mathrm{k})=1 \mathrm{y} \mathrm{k}_{\mathrm{c}}^{-1}$ $\frac{\mathrm{kT}_{\mathrm{c}}}{\mathrm{j}}=\frac{2}{\operatorname{arcoss} \mathrm{h} \sqrt{2}}=\frac{2}{\mathrm{In}(\sqrt{2+1})} \approx 2.2699$. These approximation imply that:

$$
\mathrm{K}_{1}(\mathrm{k}) \approx\left[\operatorname{In} 4\left(1-\mathrm{k}^{2}\right)^{\frac{-1}{2}}\right] \approx|\mathrm{n} 4| \frac{\partial \mathrm{K}_{1}(\mathrm{k})}{\partial \mathrm{k}} \approx \frac{\pi}{2}, \mathrm{E}_{1}(\mathrm{k})=1
$$

These results indicate that there is logarithmic divergence of the specic heat capacity close to $\mathrm{T}_{\mathrm{c}}$ :

$$
\begin{gathered}
\mathrm{C}(\mathrm{kT}) \approx \frac{2 \mathrm{k}}{\pi}\left(\frac{2 \mathrm{j}}{\mathrm{kT}_{\mathrm{c}}}\right)^{2}\left|\mathrm{n}\left(1-\frac{\mathrm{T}}{\mathrm{T}_{\mathrm{c}}}\right)+\right| \mathrm{n}\left(1-\frac{\pi}{4}\right) \\
\mathrm{C}(\mathrm{T}) \approx \frac{2 \mathrm{k}}{\pi}\left(\frac{2 \mathrm{j}}{\mathrm{kT}}\right)^{2}|\mathrm{n}| 1-\frac{\mathrm{T}}{\mathrm{T}_{\mathrm{c}}} \mid
\end{gathered}
$$

In the presence of an external magnetic field, the temperature dependence of spontaneous magnetization close to $T_{c}$ is obtained based on:

$$
\mathrm{m}=\beta \frac{\partial \mid \mathrm{nz}}{\partial \mathrm{h}}
$$

The calculation is based on the thermodynamic limit and for small fields. By Yang (1952), published a first expression for the magnetization:

$$
\mathrm{m}=\left\{\begin{array}{ll}
\left\{1-(\sinh 2 \beta \mathrm{j})^{-4}\right\}^{\frac{1}{8}}, & \mathrm{~T}_{\mathrm{c}} \leq \mathrm{T} \\
0, & \mathrm{~T}_{\mathrm{c}} \geq \mathrm{T}
\end{array}\right\}
$$

In terms of the critical point:

$$
\mathrm{m}(\mathrm{T}) \sim\left[\sinh \left(2 \beta_{\mathrm{c}} \mathrm{j}\right)^{2}-\sinh \left(2 \beta_{\mathrm{j}}\right)^{4}\right]^{\frac{1}{8}} \sim \mathrm{A}\left(\mathrm{T}_{\mathrm{c}}-\mathrm{T}\right)^{\frac{1}{8}}
$$

For susceptibility:

$$
\mathrm{X}=\frac{1}{\mathrm{~N}} \frac{\partial \mathrm{m}}{\partial \mathrm{h}}=\frac{\mathrm{kT}}{\mathrm{N}} \frac{\partial^{2} \mid \mathrm{nz}}{\partial \mathrm{h}^{2}}
$$

\section{MATERIALS AND METHODS}

Ising model simulation for $2 \mathrm{D}$ lattice for $2 \times 2$ size: The macroscopic properties of a material in thermodynamic equilibrium are usually described based on the knowledge of macroscopic properties supported by statistical laws that govern the particle array. For example, in the formalism of the canonical ensemble, the probability of finding the ensemble in a particular state with energy $\left(\mathrm{E}_{\mathrm{i}}\right)$ is given by Gibbs (1902) and Walecka (2000):

$$
p_{i}=\frac{1}{z} e^{-\frac{E_{i}}{k T}}=\frac{1}{\sum_{i} e^{-\frac{E_{i}}{k T}}} e^{-\frac{E_{i}}{k T}}=\frac{e^{-\beta E_{i}}}{\sum_{i} e^{-\beta E_{i}}}
$$

where, $\beta=1 / \mathrm{kT} \mathrm{k}$ and $\mathrm{T}$ Boltzmann's constant and absolute temperature, respectively. The expected value of an observable $(Q)$ in thermal equilibrium in the phase space (space in which all the possible micro-states of the system can be found) is given by:

$$
\langle\mathrm{Q}\rangle_{\mathrm{T}}=\sum_{\mathrm{i}} \mathrm{Q}_{\mathrm{i}} \mathrm{P}_{\mathrm{i}}=\frac{1}{\mathrm{z}} \mathrm{Q}_{\mathrm{i}} \mathrm{e}^{-\beta E_{\mathrm{i}}}
$$

For the internal energy:

$$
<\mathrm{Q}\rangle=\mathrm{E}=\frac{1}{\mathrm{z}} \sum_{\mathrm{i}} \mathrm{E}_{\mathrm{i}} \mathrm{e}^{-\beta \mathrm{E}_{\mathrm{i}}}
$$

For the observables heat capacity $(C)$, magnetization (m) and susceptibility $(\chi)$ are also expressed in terms of the partition function (Eq. 12, 17 and 20). Many algorithms have been developed to determine the value of a physical observable of a system involving long and short range interactions (emphasis is placed on discrete models). In the particular case where interaction between spins is involved (close neighbors), the Monte Carlo simulation in conjunction with the Metropolis algorithm (for a more detailed description (Sandvik, 2011, Binder, 1981, 1997; Landau and Binder, 2000; Metropolis and Ulam, 1949) is particularly relevant. The methodology has allowed us to randomly simulate some of the thermal properties of the system (thermal fluctuations). The method is designed to generate a trajectory in the phase space in which each point is a state of the system and one step at a time is taken (one step is equivalent to a transition from one state to another). The corresponding observable are calculated and stored before taking a new step. After a certain number of steps the system tends towards a state which is considered to be in thermal equilibrium. That is even when another step is taken, the system remains in the same state. Finally, an average is calculated of all the stored observable to obtain mean values. In the transit from one state to another, it is very common to use the methodology known as the Markov chain which conditions the system to always reach a state of thermal equilibrium. Markov chains (Kemeny and Snell, 1983) provide the necessary information on the probability of passing to any state in the system in the phase space, guaranteeing the transition from state to state and ensuring that a state of equilibrium is reached. The Ising model is of great utility when contextualizing the 
Metropolis algorithm in the field of spin interactions. It is used to allocate a direction to the spin moment whereas the magnetic moment is presented by the magnitude of exchange interaction. For the case of $2 \mathrm{D}$ the lattice sites can only take the value of $\mathrm{S}= \pm 1$ ( +1 for spins pointing upwards and -1 for spins pointing downwards). The Metropolis algorithm (Sandvik, 2011; Binder, 1981, 1997; Binder and Heermann, 1988; Metropolis and Ulam, 1949) traces a sequence of stochastic process that will allow a previously established configuration of spins to transit to a new configuration (a Monte Carlo Step (MCS)) that can be summarized as:

- An initial configuration of the system (for $\mathrm{N}$ lattice sites) with spin configuration $\mathrm{S}_{0}$

- A lattice site $\mathrm{m}(1<\mathrm{m}<\mathrm{N})$ is visited with spin configuration $\mathrm{S}_{\mathrm{i}}$. The spin of this site is inverted and it is associated to a configuration labeled $\mathrm{S}_{\mathrm{j}}$

- The energy shift per spin inversion is calculated that is $\Delta \mathrm{E}=\mathrm{E}_{\mathrm{Sj}}-\mathrm{E}_{\mathrm{Si}}$

- If $\Delta \mathrm{E}$ is less than or equal to zero, the inversion is accepted $\left(S_{i+1}=S_{j}\right)$ sand we go on to step 2

- If $\Delta \mathrm{E}$ is positive, a random number $\mathrm{r}(0<\mathrm{r}<1)$ is generated, if this number is lower than the transition probability, $\mathrm{S}_{\mathrm{i}+1}$ is taken as $\mathrm{S}_{\mathrm{j}}$. In other cases, the spin configuration remains unchanged. We move on to step 2 until completing the visits in all system spins

- The desired observable are calculated

An MCS is carried out for when all of the lattice points are visited. The probability to describe the system behavior is the same as the transition probability and is defined as:

$$
p\left(S_{i}-S_{j}\right)=\left\{\begin{array}{lrr}
1 & \text {,si } & \Delta E \leq 0 \\
e-\frac{\Delta E}{k T}, \text { si } & \Delta E>0
\end{array}\right\}
$$

By calculating the observable using the MCs for the magnitudes, energy, magnetization, specific heat capacity and susceptibility in relation to the temperature are obtained based on the expressions (Fluctuation Dissipation Theorem); Energy per spin:

$$
<\mathrm{E}>=\frac{1}{2}<\sum_{\mathrm{i}}^{\mathrm{N}} \mathrm{H}_{\mathrm{i}}>=\frac{1}{2}<-\mathrm{j} \sum_{\mathrm{i}}^{\mathrm{N}} \sum_{\mathrm{jnm}} \mathrm{S}_{\mathrm{i}} \mathrm{S}_{\mathrm{j}}>
$$

Specific heat capacity:

$$
\mathrm{C}=\frac{\partial \mathrm{E}}{\partial \mathrm{T}}=\frac{\left\langle(\Delta \mathrm{E})^{2}\right\rangle}{\mathrm{kT}^{2}}=\frac{\left\langle\mathrm{E}^{2}\right\rangle-\langle\mathrm{E}\rangle^{2}}{\mathrm{kT}^{2}}
$$

Magnetization:

$$
<\mathrm{m}>=\frac{1}{\mathrm{~N}} \sum_{\mathrm{i}}^{\mathrm{N}} \mathrm{S}_{\mathrm{i}}
$$

Magnetic susceptibility:

$$
\chi=\frac{1}{\mathrm{~N}} \frac{\partial \mathrm{m}}{\partial \mathrm{h}}=\frac{1}{\mathrm{NkT}}\left(<\mathrm{m}^{2}>-<\mathrm{m}>^{2}\right)
$$

The equations infer that the specific heat capacity is proportional to the fluctuations of the system's energy levels while susceptibility is proportional to fluctuations in the magnetization.

Theoretical model; The $2 \mathrm{D}$ lattice for $2 \times \mathbf{2}$ size: In order to illustrate the theoretical calculation of the observable of the magnetic system where spin interaction among immediate neighbors is involved, a $2 \times 2$ two-dimensional array has been considered. Now, the basic principles of statistical mechanics establish the possible number of configurations, defigeneration, energy and magnetization of the system, these are listed in Table 1. Arbitrary units (a.u) are used to facilitate management and comprehension. The partition function and the respecfitive observable for this particular case are as follows:

$$
\begin{aligned}
& \mathrm{z}=\sum_{i}^{4} \operatorname{Deg}(\mathrm{i}) \mathrm{e}^{-\beta H_{i}}=2 \mathrm{e}^{8 \beta}+8 \mathrm{e}^{(0) \beta}+ \\
& 4 \mathrm{e}^{(0) \beta}+2 \mathrm{e}^{-8 \beta}=2 \mathrm{e}^{8 \beta}+12+2 \mathrm{e}^{-8 \beta}
\end{aligned}
$$

\begin{tabular}{|c|c|c|c|}
\hline $\begin{array}{l}\text { Configuration } \\
\text { spin (No.) }\end{array}$ & $\begin{array}{c}\text { Degeneracy } \\
\text { (No.) }\end{array}$ & $\begin{array}{c}\text { Energy } \\
\text { (a.u) }\end{array}$ & $\begin{array}{l}\text { Magnitization } \\
\text { (a.u) }\end{array}$ \\
\hline \multicolumn{4}{|l|}{11} \\
\hline 11 & 2 & -8 & $+4,-4$ \\
\hline \multicolumn{4}{|l|}{11} \\
\hline 11 & 8 & 0 & $+2,-2$ \\
\hline \multicolumn{4}{|l|}{ \ } \\
\hline $1+$ & 4 & 0 & 0 \\
\hline \multicolumn{4}{|l|}{11} \\
\hline+1 & 2 & 8 & 0 \\
\hline
\end{tabular}

From this set of equations, we can determine the specific heat capacity and magnetic susceptibility based on the temperature:

$$
\begin{aligned}
& <\mathrm{E}>=-\frac{1}{\mathrm{Z}}\left[2(8) \mathrm{e}^{8 \beta}+2(-8) \mathrm{e}^{-8 \beta}\right] \\
& \left.<\mathrm{E}^{2}>=\frac{-1}{\mathrm{Z}}\left[2(64) \mathrm{e}^{8 \beta}+2(64) \mathrm{e}^{-8 \beta}\right)\right] \\
& <|\mathrm{m}|>=\frac{1}{\mathrm{z}}\left[2(4) \mathrm{e}^{8 \beta}+8(2)\right] \\
& <\mathrm{m}^{2}>=\frac{1}{\mathrm{z}}\left[2(16) \mathrm{e}^{8 \beta}+8(4)\right]
\end{aligned}
$$




\section{RESULTS AND DISCUSSION}

The interaction of the observable is compared (energy, magnetization, heat capacity and susceptibility) with the temperature for the models; Onsager, Ising theoretical and simulation developed in this research. Figure 1 shows the variation of magnetization per spin vs. temperature for the $2 \times 2$ planar array (2D) for the formalism's Onsager model (Eq. 18), theory model (Eq. 29) and Ising simulation (Eq. 26). The results for the three models show a better fit than $1 \%$ for the entire temperature range. For all three methodologies, they show the presence of a phase transition in the array (paramagnetic/ferromagnetic transition). By comparing the value of the transition temperature for the respective formalism, we conclude that the dispersion is close to $1 \%$ (Figure 1). This corroborates the good fit of the simulation model (Ising) with the other two models in the entire temperature range considered in this research. Figure 1 shows the variation of the heat capacity per spin vs. temperature for the $2 \times 2$ size for $2 \mathrm{D}$ lattice (Eq. 25 and 29). The maximum position of the peak is correlated to the first order transition temperature (par-ferromagnetics). Similarly, to Fig. 2 the results show a good fit for the two models. Figure 3 shows the variation of the magnetic susceptibility per spin vs. temperature for the $2 \times 2$ for $2 \mathrm{D}$ lattice. The results show the correlation of the fluctuations of the total magnetization in relation to the temperature (Eq. 27 and 29). For the case of the simulation with a small number of spins $(2 \times 2)$ if the position of the spin is chosen randomly given that there are not many options to choose from this causes a great diffierence between the data obtained depending on whether the position is chosen in an orderly or random fashion. However, when the number of spins increases, the probability of a spin being chosen more than once is reduced. This implies that random choice is increasingly like an ordered choice where a spin is only chosen once. This is further proof that in a statistical method such as the MCS, the more random all its processes, the better the results obtained. When using MCs and the MCS number, the question that emerges is whether there is such a thing as an ideal MCS number. According to the literature, the "ideal" number of steps in MCS in computational simulations of magnetic materials is around $10^{6} \mathrm{MCS}$. In most cases, this number ensures the thermal balance of the system without wasting a colossal amount of computational time. As a partial conclusion of this research, it discerns that the Ising $2 \mathrm{D}$ simulation method developed provides results of the observable that fit quite well to the theoretical model. Comparing these results with those reported in reference (Jacques, 2008), we can conclude that this methodology can opti mize the model throughout the temperature range.

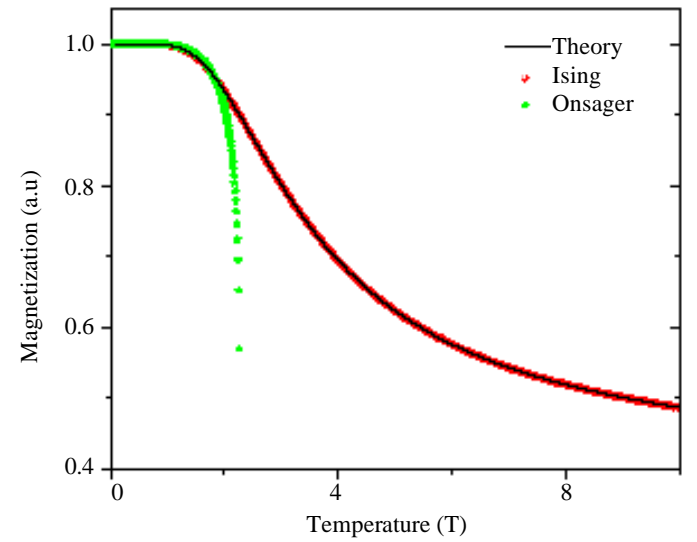

Fig. 1: Comparison between theoretical, Onsager and Ising modulation of magnetization vs. temperature for finite for $2 \mathrm{D}$ lattice of $2 \times 2$ size

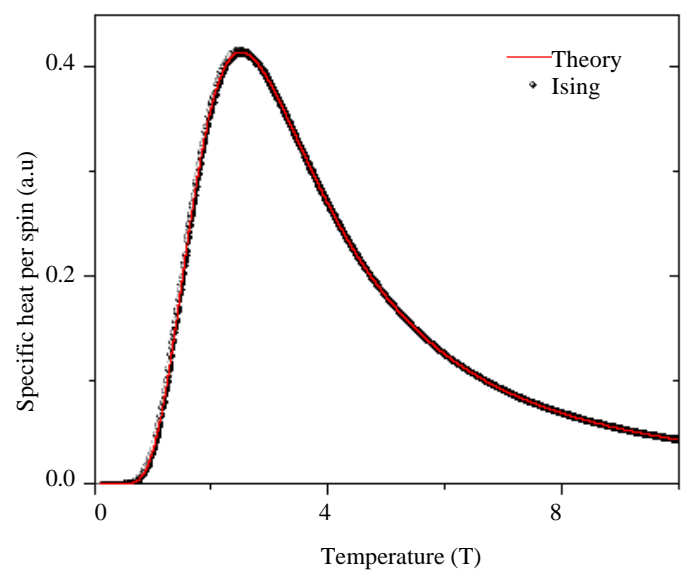

Fig. 2: Comparison between theoretical and Ising modulation of heat capacity per spin vs. temperature for finite for $2 \mathrm{D}$ lattice of $2 \times 2$ size

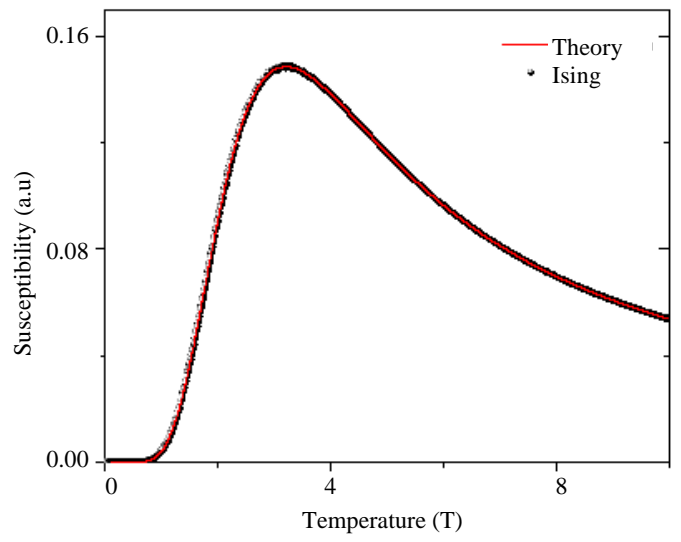

Fig. 3: Comparison between theoretical and Ising simulate of magnetic susceptibility per spin vs. temperature for finite for $2 \mathrm{D}$ lattice of $2 \times 2$ size 
This optimization result is justified by the random number generator (ran2 developed by Numerical Recipes (Binder, 1981). The observable can only be calculated when the Metropolis algorithm has been full assessed. Priority is given to calculating the observable of the states that are part of the system trajectory and not of temporal states that emerge during the evaluation of the Metropolis algorithm. The accumulated variables associated to the observable in the simulation of reference (Jacques, 2008) have a greater amount of values and many of them do not contribute relevant information and may even affect the calculation of the total average of an observable. It is obvious that the fewer the approximations made in the calculation of a magnitude, the narrower the dispersion of that magnitude. As such we have calculated the values of the energy and magnetization of the lattice at the end of each MCS. The energy of each of the nearest neighbor spins and that of the magnetization of each spin is added. The procedures in reference (Jaques, 2008) carry out the calculations through a series of approximations. For example, to calculate the energy, they use the energy from the previous lattice and add twice the difference of the energy between the current lattice and the previous lattice. On the other hand, to calculate the magnetization, they use the magnetization of the previous lattice and add twice the value of the inverted spin. It is important to note that the calculation of these observable is viable because it is carried out using the Metropolis algorithm making it possible to monitor the spins that have been inverted (Restrepo-Parra et al., 2011). Although, it is certain that the approaches may diminish the computational time in a simulation for this particular case, the form of the approaches is directly related to the moment at which they are used (within the Metropolis algorithm), bearing in mind the arguments proposed above and the loss of precision when using approximations, it is feasible to consider the difference in the way the observable are calculated as an in fluencing factor in the precision of the simulations. This argument allows us to interpret the possible discrepancy observed in the high temperature region. On the other hand, also in the high temperature region, the spins change direction more frequently which is why a greater amount of unnecessary data is obtained affecting the average of the observable. For the low temperature region, spin ordering is almost constant, leading to a better approximation of the results presented by the respective formalism. Figure 4 shows the variation of the energy per spin vs. temperature for a $2 \mathrm{D}$ lattice of different sizes (L). For all cases as the temperature tends towards zero, the energy tends towards a maximum value (approximately $-2 j=-2$ ). That is when the temperature is very close to zero, all the spins tend to

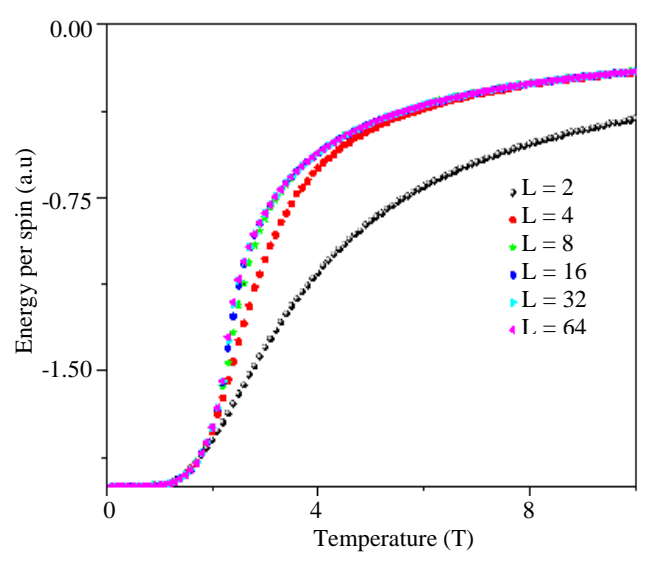

Fig. 4: Temperature dependences of the energy per spin for several lattice sizes

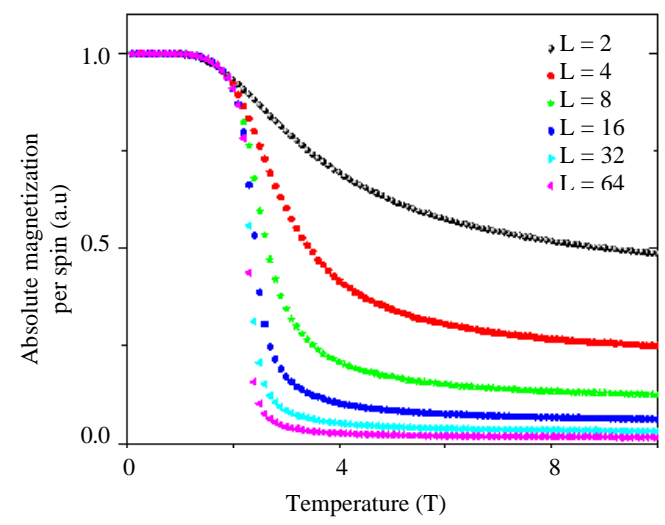

Fig. 5: Temperature dependences of the absolute magnetization per spin for several lattice sizes

align reaching the maximum value for energy in the system. For $2 \times 2$ and $4 \times 4$ sizes, respectively, the systems may involve changes in the order of spins, implicitly leading to increased energy in the region with the highest temperature. That is the spin moment is less linked as the temperature increases. This trend can also be seen in the variation of the magnetization with temperature (Fig. 5). For these lattice sizes, the transition temperature is not well defined and as size (L) increases, the dispersion in the value of the temperature diminishes. This trend is further accentuated as the heat capacity in relation to the temperature increases (Fig. 6). The trend in the temperature for magnetization, heat capacity and magnetic susceptibility with lattice size $(\mathrm{L})$, reveals that the effect of finite size scaling is present in these systems involving the spin and allowing an empirical exploration of the universality of the critical exponents.

Finite size scaling analysis: Of the magnetic response of a ferromagnetic to a temperature close to a phase 


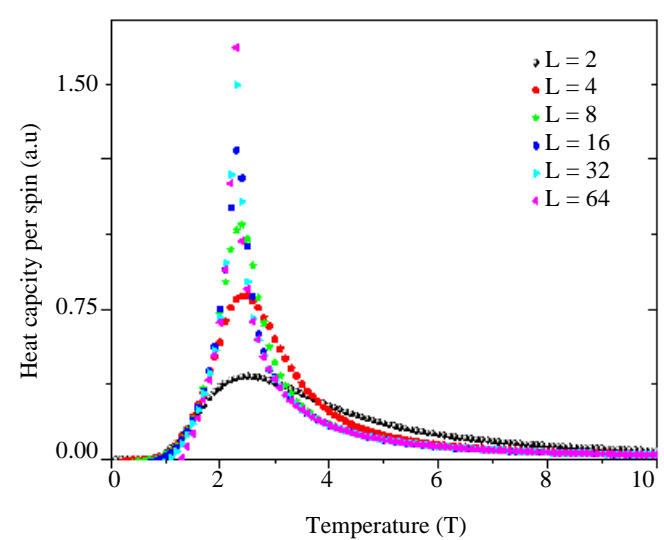

Fig. 6: Temperature dependences specifc heat capacity per spin for several lattice sizes

transition, it is very frequent based on isothermals $\mathrm{m}(\mathrm{T})$, $\chi(\mathrm{T})$ and $\mathrm{C}(\mathrm{T})$, to determine the critical exponents and $\beta, \Upsilon$, and $\alpha$, respectively. The values of the critical exponents are indicators of the class of universality in the magnetic response to thermal effects. All the systems that have the same dimensionality and the same symmetry should present the same singularities, governed by the same set of critical exponents. An example of this universality is represented in the set of equations in references (Arrott and Noakes, 1967; Newman and Berkema, 1996). Many formalism have been developed to find rigorous relationships between the critical exponents (scaling relations). Most of these are based on thermodynamic analysis. It is very common for the methodologies that calculate the value of the critical exponents to present small dispersion which are correlated to the dispersion in the precision of the value of the critical temperature. One methodology that considerably reduces this dispersion is the finite size scaling method. In the formalism, the observable energy, magnetization, heat capacity and susceptibility are known as scale functions. The scale functions are independent from lattice size but dependent on temperature and critical exponent. The model type is expressed as (Newman and Barkema, 1996, Crokidakis et al., 2009; Bhatacharjee et al., 1987; Rieger and Young, 1993; Canddau and Binder, 2002; Press et al., 1986; Ogielski, 1986):

$$
\begin{aligned}
& \xi(\mathrm{T}, \mathrm{L}) \sim\left|\mathrm{T}-\mathrm{T}_{\mathrm{c}}\right|^{-v} \rightarrow \mathrm{L} \\
& \mathrm{M}(\mathrm{T}, \mathrm{L}) \sim\left|\mathrm{T}_{\mathrm{c}}-\mathrm{T}\right|^{\beta} \rightarrow \mathrm{L}^{\frac{\beta}{v}} \\
& \mathrm{C}(\mathrm{T}, \mathrm{L}) \sim\left|\mathrm{T}_{\mathrm{c}}-\mathrm{T}\right|^{-\alpha} \rightarrow \mathrm{L}^{\frac{\alpha}{v}} \\
& \mathrm{X}(\mathrm{T}, \mathrm{L}) \sim\left|\mathrm{T}_{\mathrm{c}}-\mathrm{T}\right|^{-\gamma} \rightarrow \mathrm{L}^{\frac{\gamma}{v}}
\end{aligned}
$$

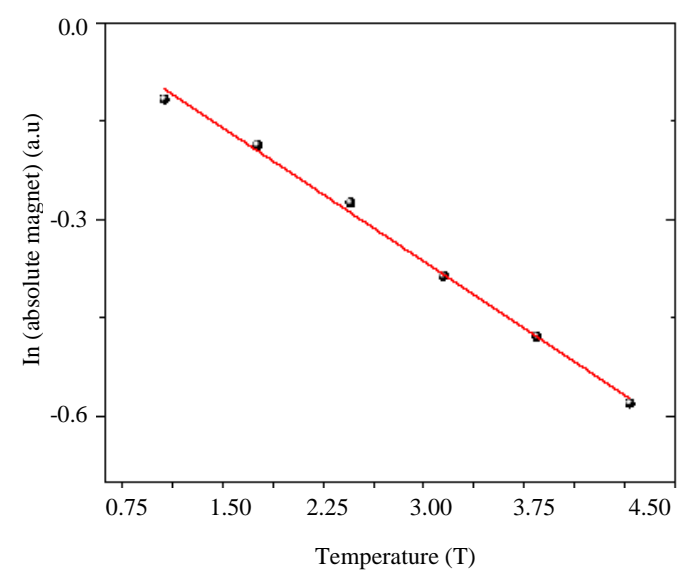

Fig. 7: Trend of the position of the maximum absolute magnetization with the size (slope $=0.13614$, standard error $=0.00425$, adj. $\mathrm{R}^{-2}=0.99514$ )

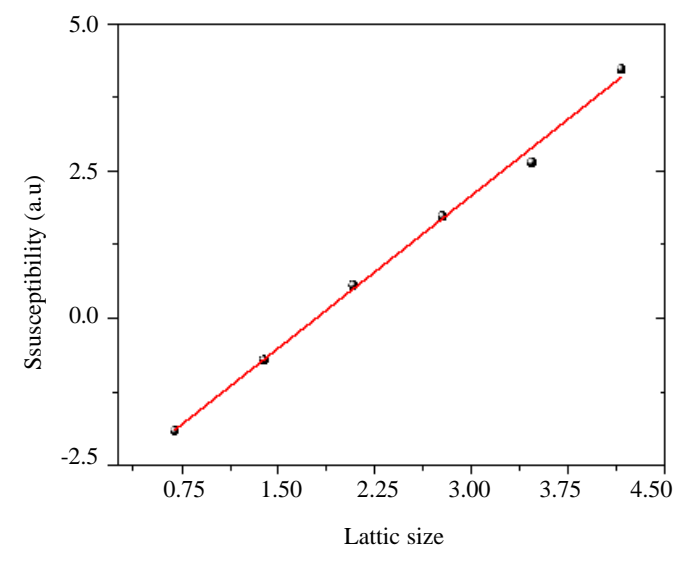

Fig. 8: Trend of the position of the maximum the susceptibility whit the size (slope $=1.72994$, standard error $=0.05006$, adj. $\mathrm{R}_{-}{ }^{2}=0.99583$ )

Exponent $v$ is related to the divergence of the longitudinal correlation $\beta$ is related to the behavior of the magnetization close to transition temperature $T_{c}, \alpha$ is related to the divergence of the specific heat capacity in $T_{c}$ and $\gamma$ refers to the divergence of the susceptibility close to the critical point. Figure 7-9 show the fit that allows the determination of the critical exponents of the observable $\mathrm{M}(\mathrm{T}, \mathrm{L}), \mathrm{C}(\mathrm{T}, \mathrm{L})$ and $\chi(\mathrm{T}, \mathrm{L})$, respectively. For the 2D Ising model, it has been established that the value of the critical exponent for the specific heat capacity is zero. However, it is frequent to plot C vs. $\ln (\mathrm{L})$ and the slope for this line is $\mathrm{C}_{0}$. The values for the critical exponents calculated in this research are compared to those reported in reference (Kote, 2008; Crokidakis et al., 2009) (Table 2). 


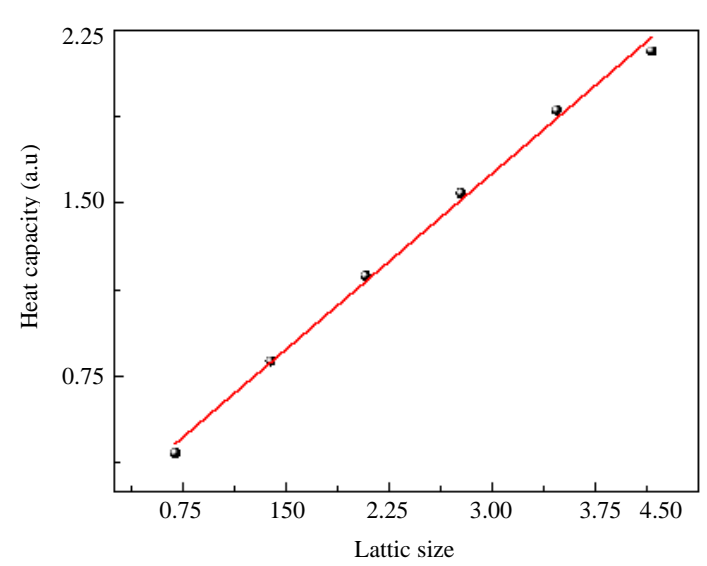

Fig. 9: Trend of the position of the maximum specific heat capacity whit the size (slope $=0.50891$, standard error $=0.01548$, adj. $R^{-2}=0.99539$ )

Table 2: Critical exponents of the observable $\mathrm{M}(\mathrm{T}, \mathrm{L}), \mathrm{C}(\mathrm{T}, \mathrm{L})$ and $\chi(T, L)$, respectively, for the $2 \mathrm{D}$ Ising model

\begin{tabular}{llllc}
\hline Magnitude & Exponent & Ref. & Simulation & Theoretical \\
\hline Magnetization & $\beta$ & $0.128 \pm 0.008$ & $0.13614 \pm 0.00425$ & 0.125 \\
Susceptibility & $\gamma$ & $1.76 \pm 0.0100$ & $1.72994 \pm 0.05006$ & 1.750 \\
Heat capacity & $\mathrm{C}_{0}$ & $0.518 \pm 0.020$ & $0.50891 \pm 0.01548$ & 0.500 \\
\hline
\end{tabular}

\section{CONCLUSION}

The 2D Ising model is a useful tool for revealing the phenomenology of magnetic materials that present phase transitions. The model provides the basic fundamentals for the computational simulation of this type of material. The computational results of MCs with the Metropolis algorithm presented in this research for a $2 \times 2$ size for $2 \mathrm{D}$ lattice showed a dispersion of close to $1 \%$ when compared to the exact solution and with the Onsager model. In terms of the curves for specific heat capacity in relation to the temperature for the $2 \times 2$ for $2 \mathrm{D}$ lattice simulated in the high temperature region, the data is much closer to the exact solution thus improving the report presented in reference (Jaques, 2008). The benefit is attributed to two factors, the first relates to the way in which the energy is calculated given that the use of approximations may affect the preciseness of the results, the second factor is the moment at which the system observable are stored and updated given that in the computational simulation proposed in the reference, this process has been carried out within the metropolis loop, leading to an accumulation of unnecessary data when the spins are inverted, affecting the average for the observable. The searguments become valid if we consider that at high temperatures, the spins are more likely to change direction and therefore, affect a greater amount of data. Another contribution of this research has to do with simulation. It can be affrmed that the choice of a suitable random number generator is not the only important factor as there are other processes involved, for example, choosing ordered or random spins which in turn, affect the value of the data obtained to a great extent in particular for small sized systems. Thus, it is important to inspect each process within the simulation and to try to implement randomness where possible. Based on the computational results for $2 \times 2,4 \times 4,8 \times 8,16 \times 16,32 \times 32$ and $64 \times 64$ for $2 \mathrm{D}$ lattices, the finite size scaling method allowed the calculation of the critical exponents of the scale functions, magnetization, specific heat capacity and susceptibility. The results showed that the scale functions are independent of network size but dependent on temperature and the critical exponent (class of universality).

\section{ACKNOWLEDGEMENT}

This research was supported by the Propiedades Termicas Dielectricas de Compositos Group Research in the Department of Basic Sciences of the Universidad Nacional de Colombia Sede Palmira, Colombia.

\section{REFERENCES}

Arrott, A. and J.E. Noakes, 1967. Approximate equation of state for nickel near its critical temperature. Phys. Rev. Lett., 19: 786-789.

Bhattacharjee, S.M., J.S. Ho and J.A.Y. Johnson, 1987. Translational invariance in critical phenomena Ising model on a quasi-lattice. J. Phys. Math. Gen., 20: 4439-4449.

Binder, K. and D.W. Heermann, 1988. Monte Carlo Simulation in Statistical Physics: An Introduction. 4th Edn, Springer, Berlin, Germany, ISBN:9783540191070, Pages: 127.

Binder, K., 1981. Finite size scaling analysis of Ising model block distribution functions. Zeitschrift Phys. Condens. Matter, 43: 119-140.

Binder, K., 1997. Applications of Monte Carlo methods to statistical physics. Rep. Prog. Phys., 60: 487-559.

Binner, J.G.P., G. Dimitrakis, D.M. Price, M. Reading and B. Vaidhyanathan, 2006. Hysteresis in the $\beta$-a phase transition in silver iodide. J. Thermal Anal. Calorim., 84: 409-412.

Brush, S.G., 1967. History of the Lenz-Ising model. Rev. Mod. Phys., 39: 883-893.

Crokidakis, N., D.O. Soares-Pinto, M.S. Reis, A.M. Souza and R.S. Sarthour et al., 2009. Finite-size analysis of a two-dimensional Ising model within a nonextensive approach. Phys. Rev., 80: 1-11. 
Gibbs, J.W., 1902. Elementary Principles in Statistical Mechanics: Developed with Especial Reference to the Rational Foundations of Thermodynamics. Charles Scribner's Sons, New York, USA., Pages: 207.

Huang, K., 1987. Introduction to Statistical Mechanics. 2nd Edn., CRC Press, Boca Raton, Florida, USA.,

Kaufman, B., 1949. Crystal statistics II: Partition function evaluated by spinor analysis. Phys. Rev., 76: 1232-1243.

Kemeny J.G. and J.L. Snell, 1983. Finite Markov Chains: with a New Appendix Generalization of a Fundamental Matrix. Springer, New York, USA., SBN-13:9780387901923, Pages: 244.

Landau D.P. and K.A. Binder, 2000. A Guide to Monte Carlo Simulations in Statistical Physics. Cambridge University Press, Cambridge, UK., ISBN-13: 9780521653664, Pages: 384.

Majumdar, C.K., 1966. Analytic properties of the onsager solution of the Ising model. Phys. Rev., 145: 158-163.

Metropolis, N. and S. Ulam, 1949. The monte carlo method. J. Am. Stat. Assoc., 44: 335-341.

Newman, M.E.J. and G.T. Barkema, 1996. Monte Carlo study of the random-field Ising model. Phys. Rev. E., 53: 393-404.

Ogielski, A.T., 1986. Integer optimization and zero-temperature fixed point in Ising random-field systems. Phys. Rev. Lett., 57: 1251-1254.
Onsager, L., 1943. Crystal statistics I: A two-dimensional model with an order-disorder transition. Phys. Rev., 65: 117-149.

Press, W.H., S.A. Teukolsky, W.T. Vetterling, B.P. Flannery and M. Met-Calf, 1986. Numerical Recipes in Fortran 90: The Art of Parallel Scientific Computing. 2nd Edn., Cambridge University Press, Cambridge, UK., ISBN-13:9780521574396, Pages: 572.

Restrepo-Parra, E., C.M. Bedoya-Hincapie, G. Orozco-Hernandez, J. Restrepo and J.F. Jurado, 2011. Monte Carlo simulation of magnetotransport

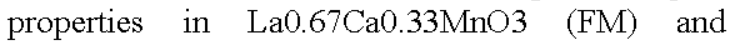
La0.33Ca0.67MnO3 (AF) thin films. IEEE. Trans. Magn., 47: 4686-4694.

Rieger, H. and A.P. Young, 1993. Critical exponents of the three-dimensional random field Ising model. J. Phys. Math. Gen., 26: 5279-5284.

Sandvik, A.W., 2011. Computational Studies of Quantumspin Systems. In: Lectures on the Physics of Strongly Correlated Systems, Avella, A. and F. Mancini, (Eds.). American Institute of Physics, College Park, Maryland, ISBN:9780735408517, pp: 335-338.

Walecka, J.D., 2000. Fundamentals of Statistical Mechanics: Manuscript and Notes of Felix Bloch. World Scientific Publishing Company, Singaporem, ISBN: 9789813102712 , Pages: 316.

Yang, C.N., 1952. The spontaneous magnetization of a two-dimensional Ising model. Phys. Rev., 85: 808-816. 